\title{
Optimal design and quantum limit for second harmonic generation in semiconductor heterostructures
}

\author{
Guido Goldoni* \\ Istituto Nazionale per la Fisica della Materia and \\ Dipartimento di Fisica, Università di Modena, Via Campi 213/A, I-41100 Modena, Italy
}

(May 9, 2019)

\begin{abstract}
The optimal design for infrared second harmonic generation (SHG) is determined for a GaAsbased quantum device using a recently developed genetic approach. Both compositional parameters and electric field are simultaneously optimized, and the quantum limit for SHG, set by the tradeoff between large dipole moments (favouring electron delocalization) and large overlaps (favouring electron localization), is determined. Optimal devices are generally obtained with an asymmetric double quantum well shape with narrow barriers and a graded region sideways to the largest well. An electric field is not found to lead to improved SHG if compositional parameters are optimized.
\end{abstract}

73.20.Dx, 78.66.Fd, 42.79.Nv

\section{INTRODUCTION}

In semiconductor quantum structures intersubband transitions lead to very large second-order optical susceptibilities 1 ; in particular, asymmetric quantum confinement may induce large second harmonic generation (SHG) in material systems, like $\mathrm{Si} / \mathrm{SiGe}$, where SHG is prohibited by symmetry in the centro-symmetric bulk host materialst; in non-centrosymmetric systems, like GaAs/AlGaAs, quantum confinement-induced SHG may exfeed by orders of magnitude the bulk contribution $\mathrm{B}$. Intersubband SHG has been intensely investigated in these and other classes of materials, like

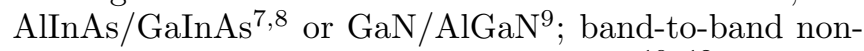
linear susceptibility has also been studied 1012

The intersubband SHG coefficient is given by

$$
\begin{aligned}
\chi_{2 \omega}^{(2)}= & \frac{q^{3}}{\epsilon_{0}} \sum_{i j} \frac{1}{2 \hbar \omega+E_{i j}-i \Gamma_{j i}} \sum_{k} \mu_{i j} \mu_{j k} \mu_{k i} \times \\
& {\left[\frac{\rho_{i}-\rho_{k}}{\hbar \omega+E_{i k}-i \Gamma_{k i}}-\frac{\rho_{k}-\rho_{j}}{\hbar \omega+E_{k j}-i \Gamma_{j k}}\right] }
\end{aligned}
$$

where $E_{l m}=E_{l}-E_{m}$ is the transition energy between subbands $l$ and $m, \mu_{l m}=\langle l|z| m\rangle$ is the dipole matrix element of the transition, $\hbar \omega$ is the photon energy, $\rho_{l}$ is the surface charge density of subband $l$, and $\Gamma_{l m}$ accounts for inhomogenous broadening of the $l \rightarrow m$ transition. Most studies of SHG focus on the double-resonant regime, where $\hbar \omega$ is in resonance with two intersubband gaps; in this regime two denominators in (11) are simultaneously resonant, and the device can be scketched as a three level system with the frequency of the incoming electromagnetic field matching approximately the $1 \rightarrow 2$ and $2 \rightarrow 3$ transitions. Hence, at the resonant frequency $\omega_{r}, \chi_{2 \omega_{r}}^{(2)}=q^{3} \rho_{1} /\left(\epsilon_{0} \Gamma^{2}\right) \xi\left(\omega_{r}\right)$, where

$$
\xi(\omega)=\frac{\mu_{12} \mu_{23} \mu_{31} \Gamma^{2}}{\left(2 \hbar \omega+E_{13}-i \Gamma\right)\left(\hbar \omega+E_{12}-i \Gamma\right)}
$$

Quantum confinement-induced SHG (i.e., in addition to the possible bulk contribution) can only be observed if the confinement potential does not have the reflection symmetry, so that none of the dipole matrix elements in (2) is zero; this can be achieved either by an asymmetrif compositione 5,13 or by application of an electric field $4416 \mathrm{~F}$ or both field, in addition to a built-in asymmetry, does not necessarily improve SHG provided that resonance is achieved by proper design of the heterostructure, so that in general the usefulness of an applied electric field from this point of view has still to be assessed.

Double-resonant SHG can be maximized by appropriately engineering the structure, in order to make the product of the three dipole matrix elements appearing in (2) as large as possible; to this end, the best compromise between large intersubband dipole moments (which favours electron delocalization) and large overlaps (which favours electron localization) must be found. The optimal design of the confinement potential, however, is a very hard optimization problem, since, in general, many compositional and geometrical parameters, and possibly the external field, can be varied, while resonance with a given radiation should be preserved in the process; furthermore, the optimal structure depends on the radiation frequency for a given class of materials. This optimization task has been undertaken so far only for specific confinement profiles; apart from the required asymmetry, the selected shapes were simple enough that optimization could be perfarmed with respect to a single parametert or the field 1 .15, 16. Analytical ontimization methods for SHG have also been proposed 17 . 18 , providing a qualitative estimate of the optimal SHG which can be obtained in a heterostructure; however, such methods could only deal with idealized continuos potential profiles, and could not include physical limitations in the alloy concentrations; for example, the optimized potentials proposed in Refs. 17,18 cannot be implemented as 
strictly type-I structures.

Recently, we have introduced a new numerical strategy which allows to optimize the performance of a given device with respect to all geometrical parameters and external fields simultaneously 19. In short, our approach uses an artificial infelligence tecnique, namely, evolutionary programming 20 , to solve the inverse problem of designing a material with preset electronic properties, including complex non-linear constraints, such as multi-subbands transition energies; by exploring the multi-dimensional parameter space, the algorithm uses a genetic paradigm to find the regions providing the best performances, and focuses the search in those regions. Using a populationbased strategy, and starting with a completely random initial set of potential profiles, the algorithm efficiently relaxes toward one or more optimal devices without limiting the search to a given class of structures. Details and demonstration of the algorithm can be found in Ref. 19. The efficiency of our algorithm allows for a quantitative modelling of the structure, including, e.g., finite band-offsets, space-dependent effective masses, band non-parabolicity, and physical limitations on alloy concentrations.

In this paper we apply our optimization strategy to determine the optimal composition for SHG from intersubband transitions in the conduction band of the GaAs/AlGaAs class of materials. We report the composition and potential profile for an optimal device with zero electric field. We also study optimal devices with a finite $F$, but we find that, in general, devices with a non-zero electric field have an estimated SHG which is weaker than devices with $F=0$.

\section{DETAILS OF THE CALCULATION}

We consider an heterostructure composed of a large number of $\mathrm{Al}_{x} \mathrm{Ga}_{1-x} \mathrm{As}$ layers, each consisting of $n$ monolayers (ML); in order to limit ourselves to type-I heterostructures, the Al molar fraction, $x$, can take continuous values in the range $[0,0.4]$; the $\mathrm{GaAs} / \mathrm{Al}_{0.4} \mathrm{Ga}_{0.6} \mathrm{As}$ band offset is large enough to accomodate the three bound states which are required to give rise to doubleresonant SHG. The structural parameters $x$ and $n$ of each layer and, in a sub-set of runs, also the eletric field $F 21$, were subjected to the evolutionary dynamics in order to maximize $\mu=\left|\mu_{12} \mu_{23} \mu_{31}\right|$ at the frequency of a $\mathrm{CO}_{2}$ laser $(\hbar \omega=0.116 \mathrm{eV})$. As mentioned above, in the doubleresonant regime the intersubband gaps match the radiation field; the possibility to implement such non-linear contraints is a distinct advantage of our numerical strategy. Since inhomogenous broadening lowers the peaks in the response function, our algorithm allows the structures to satisfy the resonance constraint only approximately; in the language of Refs. 19, 20, this is obtained using a "penalty function" with a Lorentzian shape and a width $\Gamma$ independent of the transition.
Calculations were performed within a one-band envelope function description of the conduction band singleparticle states in the low-density limit. The singleparticle Hamiltonian, with a step-like potential determined by the sequence of $\mathrm{Al}$ concentrations 22 appearing at each step of the simulation, was represented in a plane-wave basis set normalized to a periodic box; capping layers of typically $40 \mathrm{MLs}$ of $\mathrm{Al}_{0.4} \mathrm{Ga}_{0.6} \mathrm{As}$ terminate each strucure to ensure that the wavefunctions vanish at the boundaries. The space-dependent effective mass was linearly interpolated between tha GaAs and AlAs masses for arbitrary $\mathrm{Al}$ concentrations23. Non-parabolic energy dispersion is known to have a sizable effect on subband energies and wavefunction localization for large confinement energies; this is of particular concern in the design of a device for SHG, where the three bound states involved span a large energy range above the conduction band edge. By explicit calculations, of the type described below, we have found that the optimal SHG intensiy can be overestimated by $\sim 30 \%$ if non-parabolicity is neglected; in our calculations non-parabolicity effects were taken into account using an energy-dependent effective mass.24.

\section{NUMERICAL RESULTS}

The potential profiles and subband charge densities of an optimal structure, labelled $\mathbf{S 1}$, obtained with $F=0$, and a sub-optimal structure, labelled S2, with $F \neq 0$, are shown in Fig. 1. The corresponding compositional parameters are reported in Tab. 1. As can be seen from the figure, $\mathbf{S} \mathbf{1}$ has a remarkably simple shape, with sharp variations of the $\mathrm{Al}$ concentration rather than a graded composition (indeed, sub-optimal structures with a more graded composition were obtained during the simulations; see also S2). Structure $\mathbf{S 1}$ is remarkably similar to the step-graded device considered in Ref. 劧, but a) with a sharp (high and narrow) barrier between the two steps, and b) with a graded region sideways to the deeper well, which allows the charge density of the third subband to slightly delocalize out of the well region; from a large set of runs, we conclude that both these features are characteristics of the best performing structures. The calculated optimal value $\mu=3.27 \mathrm{~nm}^{3}$ of $\mathbf{S 1}$ exeeds by $37 \%$ the value $\mu=2.39 \mathrm{~nm}^{3}$ estimated in Ref. 4 .

The structure $\mathbf{S} \mathbf{2}$ is a typical example of a sub-optimal device with an optimized electric field substantially different from zero, which appeared in our simulations. The structure has a graded double quantum well shape; again, a graded region is present sideways to the largest well. As shown in Tab. 1, the estimated value $\mu=3.126 \mathrm{~nm}^{3}$ at the optimal field $F=43 \mathrm{kV} / \mathrm{cm}$, which is $\sim 4 \%$ smaller than for $\mathbf{S 1}$.

Indeed, calculations show that, in general, an electric field does not improve SHG if compositional parameters are optimized. In Fig. 2 we show the value of $\mu$ for 200 
structures which are optimized with respect to structural parameters and the electric field (open dots); the devices $\mathbf{S 1}$ and $\mathbf{S 2}$ are also indicated (solid triangles). As it is apparent, the best performing structures tend to have a small or negligible field; the possibility to achieve large SHG is substantially decreased for fields larger than approximately $60 \mathrm{kV} / \mathrm{cm}$.

Although the best performing device, $\mathbf{S 1}$, is obtained for a null electric field, an optimally designed biased heterostructuremay be desirable if field-controlled SHG is to be achieved $\mathbf{6}$. For example, $\mathbf{S 2}$ is designed to be doublyresonant at $F=43 \mathrm{kV} / \mathrm{cm}$; when the field is switched off, the intersubband gaps become $0.132 \mathrm{eV}(1 \rightarrow 2)$ and $0.103 \mathrm{eV}(2 \rightarrow 3)$, i.e., the resonance condition is lost; accordingly, the SHG drops by two orders of magnitude, although the value of $\mu$ is barely affected.

The benefit from huge non-linearities which can be obtained by quantum confinement can be limited by absorption from the material at the SHG frequencies; it has been suggested 1 that optimal conversion efficiency might be obtained with $\mu_{12} / \mu_{13} \simeq 1$. On the other hand, our simulations show that optimal quantum (i.e., confinement-induced) SHG efficiency is generally obtained with $\mu_{12} / \mu_{13} \simeq 2$ (this is, e.g., the case for devices S1 and S2), which reduces the conversion efficiency by a factor of $\sim 2$.

In summary, the present investigation of the optimal composition for double-resonant SHG in GaAs-based devices suggests that the largest quantum confinementinduced SHG is obtained in asymmetric double-quantum wells with narrow and high barriers and a graded composition sideways to the largest well. Furthermore, an electric field does not necessarily improve the SHG if structural parameters are optimized.

I acknowledge several useful discussions with Fausto Rossi.

* E-mail: goldoni@unimo.it

${ }^{1}$ E. Rosencher, P. Bois, J. Nagle, E. Costard, and S. Delaitre, Appl. Phys. Lett. 55, 1597 (1989).

${ }^{2}$ M. Seto, M. Helm, Z.Moussa, P. Boucaud, F. H. Julien, J.M. Lourtioz, J. F. Nützel, and G. Abstreiter, Appl. Phys. Lett. 65, 2969 (1994).

${ }^{3}$ P. Boucaud, F. H. Julien, D. D. Yang, and J-M. Lourtioz, E. Rosencher, P. Bois, and J. Nagle, Appl. Phys. Lett. 57, 215 (1990).

${ }^{4}$ E. Rosencher and Ph. Bois, Phys. Rev. B 44, 11315 (1991).

${ }^{5}$ W. W. Bewley, C. L. Felix, J. J. Plombon, M. S. Sherwin, M. Sundaram, P. F. Hopkins, and A. C. Gossard, Phys. Rev. B 48, 2376 (1993).

${ }^{6}$ E. Rosencher, J. Appl. Phys. 73, 1909 (1993).

${ }^{7}$ C. Sirtori, F. Capasso, D. L. Sivco, S. N. G. Chu, and A. Y. Cho, Appl. Phys. Lett. 59, 2302 (1991).
${ }^{8}$ C. Sirtori, F. Capasso, D. L. Sivco, A. L. Hutchinson, and A. Y. Cho, Appl. Phys. Lett. 60, 151 (1992).

${ }^{9}$ A. Liu, S.-L. Chuang, and C. Z. Ning, Appl. Phys. Lett. 76, 333 (2000).

10 J. Khurgin, Appl. Phys. Lett.51, 2100 (1987); Phys. Rev. B 38, 4056 (1988).

${ }^{11}$ H. Kuwatsuka and H. Ishikawa, Phys. Rev. B 50, 5323 (1994).

12 T. Park, G. Gumbs, and Y. C. Chen, J. Appl. Phys. 86, 1467 (1999).

${ }^{13}$ M. J. Shaw, K. B. Wong, and M. Jaros, Phys. Rev. B 48, 2001 (1993).

${ }^{14}$ M. M. Fejer, S. J. B. Yoo, R. L. Byer, A. Harwit, and J. S. Harris, Jr., Phys. Rev. Lett. 62, 1041 (1989).

${ }^{15}$ L. Tsang, E. Ahn, and S. L. Chuang, Appl. Phys. Lett. 52, 697 (1988).

${ }^{16}$ L. Tsang and S. L. Chuang, Appl. Phys. Lett. 60, 2543 (1992).

${ }^{17}$ S. Tomić, V. Milanović, and Z. Ikonić, Phys. Rev. B 56, 1033 (1997).

18 S. Tomić, V. Milanović, and Z. Ikonić, J. Phys.: Condens. Matter 10, 6523 (1998).

${ }^{19}$ G. Goldoni and F. Rossi, Optics Lett. 25, 1025 (2000).

${ }^{20}$ Z. Michalewicz, Genetic Algorithms + Data Structures = Evolution Programs (Springer-Verlag, Berlin, 1992); D. E. Goldberg, Genetic Algorithms in Search, Optimization, and Machine Learning, Addison-Wesley (1989); M. Gen and R. Cheng, Genetic algorithms \& Engineering Design (John Wiley \& Sons, Inc., New York, 1997).

21 The alghoritm described in Ref. 19 has been modified to include the field in the evolutionary dynamics.

22 The band gaps are chosen as follows: the $\mathrm{Al}_{x} \mathrm{Ga}_{1-x} \mathrm{As}$ band-gap $E_{g}(x)$ is obtained from $E_{g}(x)=E_{g}^{\mathrm{GaAs}}+1.36 x+$ $0.22 x^{2}$ [C. Bosio et al., Phys. Rev. B 38, 3263 (1988)]; the conduction band offset is obtained subtracting the valence band contribution $\Delta E_{v}(x)=0.48 x[\mathrm{E}$. T. Yu et al., Solid State Phys. 46, 2 (1992)].

23 The space-dependent effective mass is $m_{e}(x)=0.067+$ 0.083x [Landolt-Börnstein, Semiconductors: physics of goup IV elements, and III-V compunds, O. Madelung, Ed. (Springer-Verlag, Berlin, 1982), vol. 17].

${ }^{24}$ We choose $m_{e}(x, E)=m_{e}(x)\left[1+(E-U(x)) / E_{g}(x)\right]$ for $E>U(x)$ and $m_{e}(x, E)=m_{e}(x)\left[1+(U(x)-E) / E_{g}(x)\right]$ for $E<U(x)$ [D. F. Nelson, R. C. Miller, and D. A. Kleinman, Phys. Rev. B 35, 7770 (1987)]. 
TABLE I. Compositional parameters for the optimized devices $\mathbf{S 1}$ and $\mathbf{S 2}$. S1 is obtained with $F=0$, while $\mathbf{S 2}$ is obtained including $F$ in the optimization. For each structure, the width of each layer, $n$ (in $\mathrm{ML}$ ), and the corresponding $\mathrm{Al}$ concentration, $x$ is reported; two capping layers with $x=0.4$ enclose each structure. Inter-subband dipole moments, $\mu_{i j}$, and $\mu=\left|\mu_{12} \mu_{23} \mu_{31}\right|$ are also reported in the table. Calculations have been performed with $\hbar \omega=0.116 \mathrm{eV}$ and $\Gamma=7 \mathrm{meV}$; the resulting inter-subband gaps match $\hbar \omega$ within $1 \mathrm{meV}$. The correponding potential profiles and subband charge densities are shown in Fig. 1.

\begin{tabular}{|c|c|c|c|c|c|c|c|c|c|c|}
\hline S1 & $(F=0)$ & & & & & & & & & \\
\hline$\overline{n:}$ & (cap layer) & 6 & 12 & 2 & 16 & 6 & 6 & (cap layer) & & \\
\hline$x:$ & 0.400 & 0.149 & 0.130 & 0.363 & 0.000 & 0.286 & 0.393 & 0.400 & & \\
\hline \multicolumn{11}{|c|}{$\mu=3.27 \mathrm{~nm}^{3} \quad \mu_{12}=1.61 \mathrm{~nm}$} \\
\hline \multicolumn{11}{|c|}{$\begin{array}{ll}\mathbf{S 2} & (F=43 \mathrm{kV} / \mathrm{cm})\end{array}$} \\
\hline$\overline{n:}$ & (cap layer) & 4 & 6 & 4 & 14 & 4 & 4 & 4 & 6 & (cap layer) \\
\hline$x:$ & 0.400 & 0.385 & 0.328 & 0.203 & 0.000 & 0.209 & 0.195 & 0.143 & 0.121 & 0.400 \\
\hline \multicolumn{2}{|c|}{$\mu=3.13 \mathrm{~nm}^{3}$} & $\mu_{12}=1.63 \mathrm{~nm}$ & $\mu_{23}$ & $2.38 \mathrm{~nm}$ & $\mu_{31}=$ & $81 \mathrm{~nm}$ & & & & \\
\hline
\end{tabular}
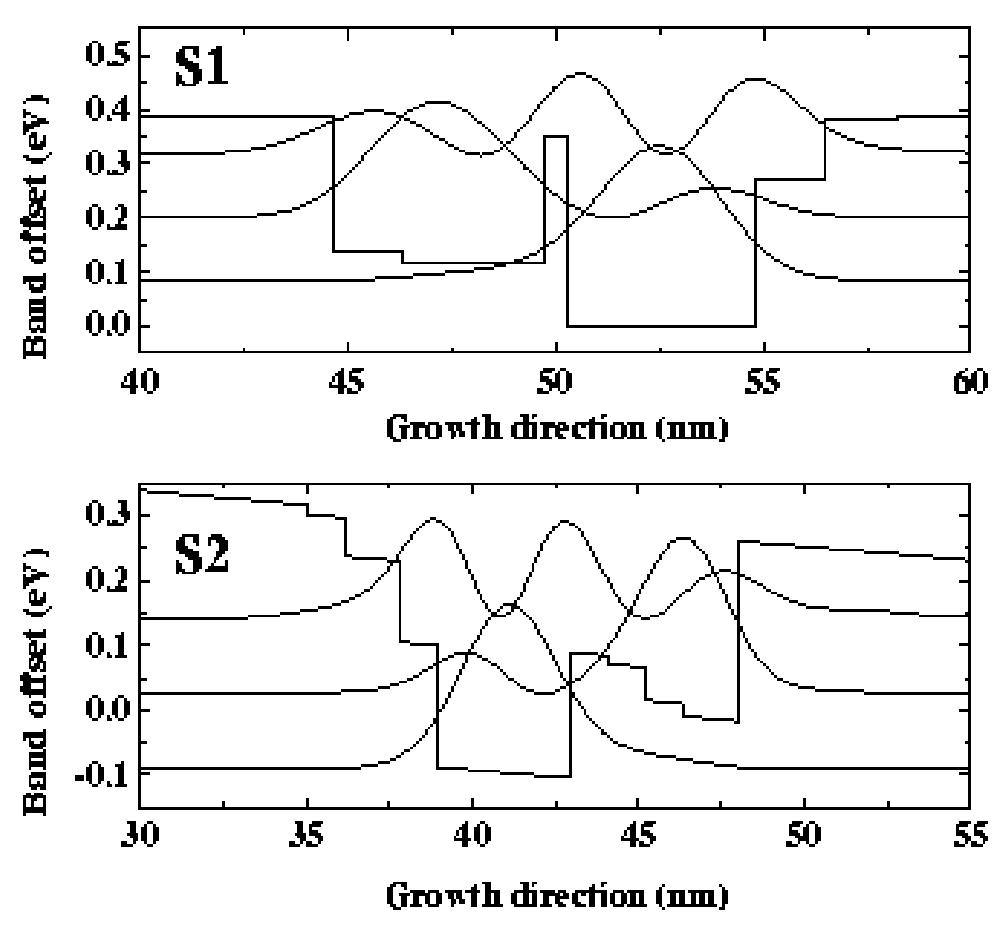

FIG. 1. Potential profiles of the optimized devices S1 and S2; the corresponding compositions are detailed in Tab. 1. Also shown are the charge densities of the three bound states involved in the SHG, vertically shifted by the confinement energy. 


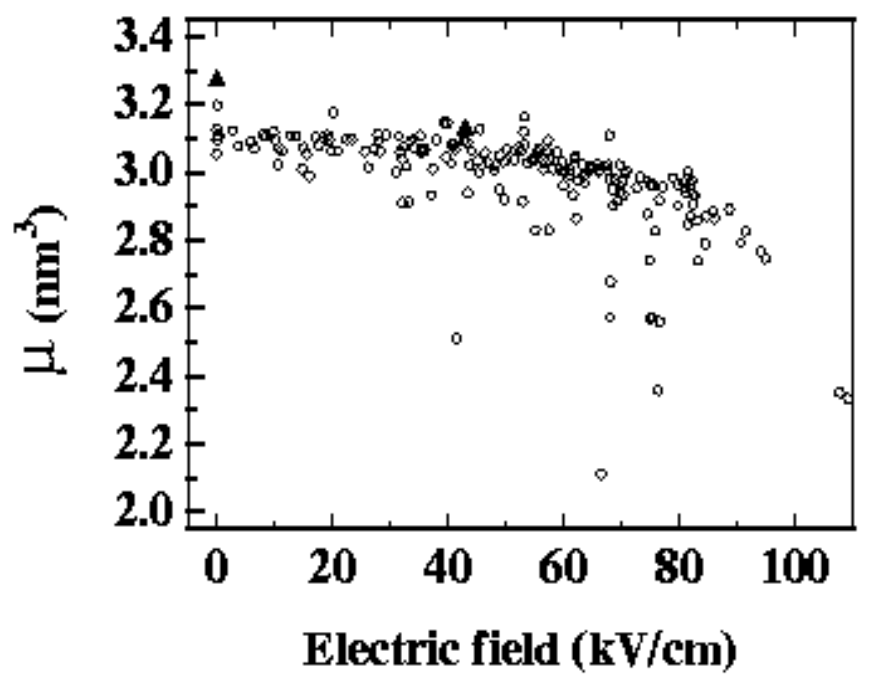

FIG. 2. Dipole moment $\mu$ vs electric field for a set of 200 devices (open dots) which have been optimized by evolutionary dynamics with respect to compositional parameters and electric field. Also shown are the values corresponding to devices $\mathbf{S 1}$ and S2 (full triangles). 